\title{
Grundtvig und Kierkegaard als Grundleger eines modernen sozialen Bewusstseins
}

\author{
von Christa Kühnhold
}

Die folgenden Ausfuhrungen halten sich eng an das im Titel vorgegebene Thema: Wie haben Grundtvig und Kierkegaard es vermocht, ein neues soziales Bewusstsein zu entwickeln, dessen Gültigkeit auch heute noch nicht überholt ist? Bewusst musste aus Zeitgründen darauf verzichtet werden, über das Thema hinaus direkte Verbindungen beider Denker zum modernen "gennembrud", die in grosser Zahl zu entwickeln wären, aufzuzeigen.

Trotz allgemein noch anhaltender a-historischer Tendenz unserer Wissenschaft, die sich literatur-historisch dünkt, wenn sie nur 200 Jahre zurückblickt, möchte ich hier, wie schon oft, behaupten, dass wir unsere Gegenwart nicht verstehen werden, wenn wir nicht die Gedanken unserer Vergangenheit denken lernen. Und zwar auch jene Gedanken, die weiter als zweihundert Jahre zurückliegen. Und wir werden die jüngere Vergangenheit z.B. "det moderne gennembrud " nicht erkennen und verstehen lernen, nicht ahnen wie er strukturiert ist, ohne die Gedanken, die ihn vorbereitet und geformt haben, genau zu kennen, Gedanken, die ihm all sein Material geboten haben.

Ein Durchbruch kommt nicht plötzlich und von ungefähr; schon lange bereitet er sich vor. Ein "Durchbruch " impliziert ein längeres, wiederholtes Anstürmen gegen Bollwerke, solange, bis dann endlich die Dämme brechen. Es stellt sich dann die Frage, woher diese "bahnbrechenden" Ideen kamen und wer ihre Wegbereiter waren. Wie werden nun die Hauptpunkte in dem Werk der Wegbereiter, der Bahnbrecher in ihrem Wirken und Schaffen angelegt sein, und wie entwickeln sie sich?

Ein Vortrag in der XVI Studien-Konferenz der IASS (International Association for Scandinavian Studies) über den modernen Durchbruch in skandinavischer Literatur gehalten (Göteborg Universitet, August 1986). Der Vortrag wurde an der Universität in Greifswald 1986 wiederholt 
Die beiden wirksamsten Kritiker ihrer Zeit, ja des gesamten 19. Jahrhunderts, in Dänemark waren, weil ihre Wirkung bis in unsere Zeit nicht abgeschlossen und nicht abzuschätzen ist, N.F. S. Grundtvig und Sören Kierkegaard. Doch wie wirkten diese beiden Kämpfer, die ihr Leben lang nichts anderes getan haben, als mit dem Einsatz ihrer gesamten Existenz gegen verstockte Sprache, verstockte Kirche, verstockten Staat und die dahinter sich verbergenden Menschen und deren Ideologie anzukämpfen?

1. Man hält den Atem an, wenn man sich vor Augen führt, was hier in der ersten Hälfte des 19. Jahrhunderts geschieht. Auf der einen Seite, von Grundtvig ausgehend, der Ruf nach einem neuen Kulturprogramm: Freiheit für den Menschen (auch für die Frauen), Freiheit von der Kirche, für die Religion, Freiheit für die Wissenschaft, für die Pädagogik, Freiheit im politischen, ökonomischen und demokratischen Sinn, "Frihed til at være sin Tro bekiendt, naar man har nogen, og sin Vantro med, naar man ingen Tro har, Frihed til at bruge Haand og Fod som man vil, lige Adgang til enhver Stilling og Syssel, naar man paa hvilkensomhelst Maade viser, at man duer til den, og alt saadant, som man veed altid er til falles Bedste." (Danskeren, August 1848, s. 373 ff.)

"Frihed for Alt, hvad der stammer fra Aand

Som ikke ændres, men arges ved Baand,

Virker skinbundet det Værste i Løn,

Tæmmes alene ved Thor-Karmens Døn!«

(Nordens Mytologi, 1832)

Hier sagt Grundtvig in aller Deutlichkeit: Wenn etwas dem Menschen schadet und ihn verdirbt, dann ist es die Unfreiheit. Und nur Donner und Blitz d.h. Kampf kann von Zwang befreien.

Grundtvigs Freund Sofus Høgsbro formulierte Grundtvigs Forderungen kurz: "Dem gemeinen Manne, d.h. den Bauern, Einsicht in das zu verschaffen was dem Wohl des Ganzen dient. "Dem Wohl des Ganzen dient die Aufklärung über Unfreiheit, Abhängigkeit und Unmündigkeit. Grundtvigs Konzeption der Volkshochschule führte nach $1864 \mathrm{zu}$ der rettenden und durch die folgenden Verfassungskämpfe nicht zerstörten geistigen Begründung der dänischen $\mathrm{Na}-$ tion.

Die Bauern, in der "Venstre" zusammengeschlossen, führten 
während der Krise der achtziger Jahre ihre ökonomische und soziale Revolution durch: die Landwirtschaft wurde auf Intensivbau und Veredelungsbetriebe für Molkerei- und Schlächtereierzeugnisse umgestellt, und zwar in eigenen Genossenschaftsbetrieben der Bauern. Kulturell hatte ihr Aufstieg durch Fortbildung in der Volkshochschule begonnen.

Fragen wir nun, warum für Grundtvig die Geschichte, die nordgermanisch-dänische Vergangenheit des Landes so wichtig geworden ist, so stossen wir auf den vorhin genannten, alles Neue begründenden Begriff der Freiheit als Zentrum seines Denkens. In Grundtvigs Jugend waren die Bauern noch unfrei. In seinen späteren Jahren gab die absolutte Monarchie, die noch fest im Sattel sass, nur sehr sparsam einige einzelne Rechte an die Bauern ab. Grundtvig nun wies mit den Hauptbegriffen "ord " und "maal" auf die schöpferische Gemeinschaft aller Menschen hin, und wollte diese Gemeinschaft im Handeln des staatlich organisierten Volkes verwirklicht sehen.

Die Grundlage solchen Handelns sah er durch das im Zusammenwirken der Menschen bedingte Wachstum der gemeinsamen Sprache gegeben. Er sagte in einer seiner Reichstagsreden: "at give hele Folkeungdommen uden Hensyn til Stand og Syssel Adgang til at faa en bedre Grund i Modersmaalet og en bedre Oplysning om Fædrelandet og alt hvad dansk er end der hidtil har været nogen offentlig Mulighed til her i Landet at erhverve til at tjene det fælles Bedste til Folkets Gavn." (Rigsdagstidende 1848-49, spalte $433 \mathrm{f}$.)

Er begegnete nur Unverständnis. Madvig antwortete: "Regeringen vil ikke vide af en særskilt, Characteren af Danskhed monopoliserende Undervisningsanstalt. " Das war eine glatte Verleugnung der Pläne Grundtvigs. Diese umfassten »Fædrelandets, altsaa gamle Danmarks Sprog, Historie, Statistik og Statsforfatning, de for Borgeren i private Stillinger vigtigste Dele af Lovgivning, og de administrative og communale Forhold. " (Forordet til "Lykønskning til Danmark..."s. 3). Es war also eine "Samfundsskole", die Grundtvig anstrebte. Der König selbst war auf seiner Seite gewesen.

Das restriktive Element der auf politischer und ökonomischer Abhängigkeit beruhenden Rangordnungen hatte das sprachliche Eigenleben Dänemarks in die Dorfgemeinschaften auf dem Lande 
zurückgedrängt. Es ist das Verdienst Grundtvigs und seiner Volkshochschulen, durch ihre Bindung an die Quellen des nordischen Altertums und Mittelalters, der von Kirche, Wissenschaft und Wirtschaft ausgetrockneten Sprache und damit dem dänischen Geistesleben, vor allem dem politischen Bewusstsein der untersten Schichten, neue Kräfte zugeführt zu haben.

"Det er i sin Orden, at jeg er udraabt for halvgal og betragtes af den latinske Minister som en Taabe, fordi jeg vil have en fælles dansk Oplysning i Landet der skal agtes langt høiere end den Oplysning og Dannelse i en enkelt Retning, som Enkeltmanden kan have Lyst og Anlæg til; thi for Latinerne gives der kun to dannede Folkefærd, som begge ere døde for længe siden, nemlig Grækere og Romere. Alle andre Folkefærd ere for dem som for Romerne Barbarer" (Danskeren, 20. dec. 1848 s. 636 f.).

Man müsste sogar von einer Bernstorff-Grundtvigschen Reform sprechen, die die kulturrevolutionären Situationen, wie sie sich in Ländern mit unterdrückten Nationalsprachen (z.B. bei den Basken und Bretonen) einstellen mussten, verhindert hat.

Wie könnte man glauben, dass Grundtvig es nicht als sein selbstverständlichstes Tun ansehen sollte, sich zum Reichstag zu begeben und dort lautstark seine Forderungen zu stellen - durch viele Jahrzehnte hindurch. Er musste Politiker werden und er wurde es, als einer der ersten Mitglieder der "grundlovsgivende Rigsforsamling " und als wortgewaltigster, unerbittlicher Kämpfer im Parlament. Für Grundtvig galt es, was unten näher ausgeführt wird, das Bild des germanischen Thingbauern, der offen, frei und selbstbewusst seine Meinung sagen konnte, in seine Zeit zu transponieren. "Jeder Mensch", dieser von Kierkegaard hundertfach gebrauchte Begriff, sollte in der Lage sein, sein Anliegen, sein Recht (altnord. ping) selbstbewusst zu vertreten.

Der selbstbewusste Mensch in Freiheit, das ist sein Ziel, und man hört es in dem Grundsatz: "Altid at unde Næsten samme Frihed, som man selv gør Krav paa. " Er war aus eigener christlicher Lebensanschauung bereit, mit jedem politischen und ideologischen Gegner zusammenzuarbeiten. "Det er den højere christelige Anskuelse... der har givet den nye Folke-Verdens TankeGang, Dannelse og Vidskab det universal-menneskelige Præg, som den gamle Verden fattedes, " og det er derfor "den eneste ægte Grund-Anskuelse af Menneske-Livet. " Diese Grundanschauung 
beruht auf dem allein christlichen Gedanken der Gleichheit aller Menschen vor Gott. Des Menschen, der zur Vollkommenheit geboren wurde (skabt i Guds billede). Hier liegt für Grundtvig der Grund für die bisher nicht übertroffene Utopie, für den Traum vom Menschen der Zukunft. Er kämpft für den freien, selbstbewussten Menschen, der frei von allem Zwang, auch frei von Neid, Missgunst und Unterdrückungssucht ist, die ihm Gewalt über andere Menschen versprechen. Ein Mensch, frei von Zwängen braucht keine Gewalt über andere. Ein Blick in die neueste tiefenpsychologische Forschung, die nach unserer historischen Kenntnis nun so neu nicht scheint, bestätigt Grundtvigs Erkenntnis. Diese höchst ursprünglich christliche und ganz gewiss nicht kirchliche "Anskuelse" Grundtvigs vom Menschenleben bietet die Geschichte als auch die tägliche Erfahrung. (Værker i Udvalg III, s. 25-27). Darum, meinte Grundtvig, sei es notwendig, der alleruntersten sozialen Schicht die Werkzeuge zu ihrer Befreiung in die Hand zu geben, nämlich Aufklärung und Wissen.

Praktisch und politisch wie Grundtvig im Gegensatz zu Kierkegaard war, notierte er bereits als ganz junger Mann 1804 in seinem Tagebuch (über Schlez: Geschichte des Dörfleins Traubenheim): "det vakte nyt Mod hos mig til engang $\mathrm{i}$ Tiden ogsaa at opbyde alt for Bønders Opklaring. Det vakte Harme og Uvillie mod alle de Bondeplagere, der existere, og især mod dem, som omringe mig, hvoraf kun faa have Religjøsitet - og da er den falsk - men ingen Moralitet. " (Værker i Udvalg I, s. 39).

Grundtvigs gesamtes Werk und Wirken, ja seit dem Beginn seines Denkens als kleiner Schüler, galt der offenen energischen Kritik am Falschen, Verbogenen, Nicht-Stimmigen, der Kritik an seiner eigenen Zeit.

Sein Leben war ein einziger Protest gegen die herrschenden Denkweisen der Epoche (sichtbar u.a. in seiner Haltung den Frauen gegenüber, z.B. Mathilde Fibiger, die in seinem Hause wohnte), die von allen ihn umgebenden Vorstellungen abwich.

Das einzige Mittel jedoch, die für ihn ekelerregende Verderbnis seiner Zeit, die sich in Unterdrückung, Hochmut, Falschheit, Profitmacherei spiegelte, zu zähmen, sah er, und das kann nicht oft genug betont werden, in ihrer Befreiung vom Zwang. So leitete er eine freie, wissenschaftliche, politische und pädagogische Diskussion ein. 
Er war es auch, der immer seinen Gegner als Menschen anerkannte, was in dem Gedicht "Menneske først og Christen saa, kun det er Livets Orden" deutlich ausgesprochen wird. Die Anerkennung dieser seiner menschlichen Haltung, hat, wohl für jeden einsichtig, die Duldung mancher späteren "Freiheiten " politischer, agitatorischer und sonstiger Art erst ermöglicht.

Grundtvig wurde von niemandem übersehen und konnte von niemandem übersehen werden:

Frihed lad være vort Løsen i Nord Frihed for Loke saavel som for Thor.

Grundtvigs soziales Programm ist in seinem Gedicht "Kirken og Skolen « klar festgelegt. Ich erlaube mir, die bezeichnenden Verszeilen zu zitieren.

1) Ordet frit i hver en Mund (Str. 9) som den tør forsvare

2) Øret frit paa samme grund Vende sig fra Mund til Mund. Hvor det bedst kan høre!

3) Bogen fri i hver Mands Haand (Str. 10) som den vil oplade

4) Livet frit for Kirke-Tugt (Str. 11) Som er ei i Ordet.

5) Derfor bort med alle Baand (Str. 7)

Es lässt sich hier die Frage stellen, in welcher Weise wohl, fast 40 Jahre später, aggressiven und z.T. hasserfüllten Proklamationen begegnet worden wäre, wenn Grundtvig nicht 1836 schon für Pressefreiheit, Meinungsfreiheit, Freiheit von Zwang der Kirche, mit dem Einsatz seiner Existens unablässig, bewusst, risikobereit und keine Verachtung scheuend (die ihm reichlich von allen Seiten zuteil wurde) gekämpft hätte.

Immer nimmt der Begriff des "lebendigen Wortes « bei Grundtvig die zentrale Stellung als Brennglas seiner Gedankengänge ein. Als le- 
bendiges Wort kommen für Grundtvig nur die Volkssprachen in Betracht, die alle Schichten des Volkes umfassen. Die Volkssprachen nähren das Geistesleben aller Volksschichten und werden von ihnen genährt. Dies Geben und Nehmen in der Sprachbewegung, die unendlich zahlreichen Akte des Geisteslebens, die mit jeder Sprachäusserung verknüpft sind, hinterlassen nachwirkende $\mathrm{Ab}-$ drücke im Sprachgebrauch. Sprache ist ein gesamt-soziales Phänomen. Das heisst die menschliche Rede wird strukturell von Elementen des historisch-traditiven Daseins und dem situativen Erlebnischarakter geprägt. Dieses Wesen der Sprache wird im Dänischen und dadurch auch von Grundtvig mit "maal " und im Hinblick auf die Verwurzelung des einzelnen Sprechers, in der Familie, als "modersmaal " bezeichnet.

Wir können nicht umhin, diese zentralen Begriffe des grundtvigschen Denkens "ord " und " maal " näher zu beleuchten, da beide die entscheidende Funktion in seiner Vorstellung von "samfund " und von der Beziehung des vom Zwang befreiten Einzelnen zu diese Gemeinschaft einnehmen.

Im Wort "maal" liegt eine wichtige Nuance des weiteren Begriffes Sprache.

"Sprog" ist die Sprache der Lehr- und Wörterbücher, auch der "toten" Sprachen. Das Wort "maal " dagegen bedeutet Stimme, Rede, Sprache, verweist also mit seiner ersten Bedeutung auf das, was jede Schrift beiseite lässt; mit der Bedeutung "Rede" verweist es auf das Lebendige des Miteinander-Sprechens und erst mit der dritten Bedeutung auf die mehr oder weniger starren Ausprägungen der Sprache, z.B. Mundarten und Standessprachen.

Dieser Grundzug im Wort "maal ", der auf die Ganzheit der Rede bei dem Sprachgebrauch hinweist, ist tief in der Geschichte des Wortes verankert. Maal über ${ }^{\star}$ mahla auf älteres germ. ${ }^{\star}$ mapla zurückgehend, stellt sich zu got. mapl, Versammlungsplatz, mapljan reden ahd. mahal "Gerichtsversammlung ".

Wie man sieht, geht die Bedeutung "Rede, Sprache" auf das Auftreten vor Gericht zurück. Das Gericht ist der Ort, an dem der freie Mann, frei seine Sache (Ding) vertreten konnte und an dem die Versammlung (samfund) ihre Meinungen und Standpunkte austauschte und mitteilte.

Hier sind alle Bevölkerungsschichten versammelt, das Miteinander wird durch Rede konstituiert, hier findet Kommunikation 
statt. Wer verstanden hat, was maal heisst, hat begriffen, was, wie Grundtvig sagt, die "Sprache unserer Väter « konstituierte.

Das Interesse des einzelnen, freien, sich seiner selbst bewussten Bauern wird beschüzt und bewahrt in der konstitutiven Volksversammlung des Things, die durch ihre Gesetzes- und Kultreden den Stamm eint! Hier wird deutlich, wie zwei Grundantriebe des menschlichen Geisteslebens: die Ehrwürde der Tradition der Väter und die Wettkampfsituation im Thinggeschehen, zusammenwirken, um Altes und Neues zur lebendigen Einheit, als dauernde Vorbilder zu prägen.

Grundtvig als intimer Kenner dieser historischen Zusammenhänge sagt: "Det er kun gjennem Folkenes Modersmaal, at Menneske-Ordet er i levende Virksomhed " (Den christelige BørneLærdom, VU VI 108 f.), "og at ethvert Tungemaals levende Udvikling i aandelig Retning kun gaar for sig under en tilsvarende Udvikling af Folkelivet i det Hele."

Die Bedeutung von maal, ord und frihed für den einzelnen Menschen ergibt sich aus diesen Formulierungen bei Grundtvig selbst.

Das Bindende zwischen den freien Einzelnen und der Gemeinschaft ist das Wort maalet.

Die entscheidende Bedeutung, die Grundtvig dem Wort, der Sprache für die soziale Freiheit und Einheit zumass, war darin begründet, dass sie ihrer Natur nach Bindungen schafft und Kraft stärkt, d.h. das Leben fördert. Diese Einsicht hatte Grundtvig sich in seinen umfassenden und gründlichen philologischen Studien erworben und diese Einsicht teilte er seiner Zeit mit. Hier, mit dem zentralen Gewicht seines Denkens, steht er bahnbrechend auf der Seite des Volkes, der Unfreien. Im unermüdlichem Kampf für ihre Würde und ihre Freiheit.

Grundtvig und Kierkegaard sind es, die in theoretischen Abhandlungen zum ersten Mal Worte und Wendungen aus der Sprache des einfachen Mannes übernehmen und auch schreiben, wie man dänisch spricht.

In der Frage nach dem "Wer" und dem "Wie" hinter dem "Was" der Dinge und Begebenheiten, wollte Grundtvig (wie auch Kierkegaard) die inhumane Unbezogenheit der willkürlichen Gesellschafts-, Verwaltungs- und Wissenschaftssprache in eine auf 
jeden einzelnen Menschen, als die jede Wirklichkeit erzeugende Kraft bezogene Sprache überführen.

Um des Lebens der Menschen willen, um unserer wirklichen Menschlichkeit willen, die den anderen nicht, wie bisher (und immer weiterhin, können wir getrost hinzufügen) als Informationsquelle, als profitförderndes Mittel zum Zweck missbraucht, sondern ihn in seiner Menschlichkeit zu fördern sucht, braucht es eine diesem neuen wirklich menschlichen Handeln angemessene Sprache, nämlich die erfahrungs- und geschichtsgesättigte Muttersprache. Die Mutter-Sprache muss um der Selbstständigkeit und der Würde jedes Menschen willen auch jedem Einzelnen zugänglich gemacht werden. Aus dieser Notwendigkeit heraus entspringt die Idee der Volkshochschule.

Grundtvigs Kampf gegen die "pluddervælske" Abstraktion der Wissenschaft wird einzig aus der Sorge um die Menschen geboren, mit denen er zusammen aufgewachsen ist. Den Bauernburschen, die nicht die Gelegenheit hatten, ihren Kopf, abstrakt, in Lateinschulen abrichten zu lassen und deshalb ausgeschlossen waren. Ausgeschlossen von allem. Aus dem Kreis derer, die sie dirigierten, manipulierten und ausbeuteten.

Deshalb begann Grundtvig seinen Kampf gegen den Idealismus, dessen Abstraktion vom einzelnen Menschen, indem er sich gegen Schelling wandte, wie Kierkegaard es in seinem Kampf gegen Hegel tat. Es gilt die "lebendige Wirksamkeit" der Volkssprache zu entfalten, die jeden Einzelnen erreicht und die ihn positiv fordern soll.

Deshalb ist es notwendig, aus der Erfahrung des Menschengeschlechtes, der Geschichte und damit aus der Geschichte der Sprache zu schöpfen. Es gilt, klar die sichere Verbindung zwischen der Erfahrung des Menschengeschlechtes und der Erfahrung des Einzelnen zu zeigen. (Om Videnskabeligheds Forhold til Erfaring og sund Menneskeforstand, s. 38).

Deshalb richten Grundtvig und auch später Kierkegaard ihre schärfsten Waffen gegen die inhaltsleeren Pseudo-Sprachen der Wissenschaft, für beide verkörpert in der Sprache der idealistischen Philosophie, und deren hybriden Anspruch Humanes allgemein und vollgültig zu repräsentieren.

Sie kämpfen gegen die Philosophie ihrer Zeit, weil sie in ihr die vernichtende Gleichgültigkeit gegen den Einzelnen, "den menige 
mand", erkennen. Eine Gleichgültigkeit, die durch "schimärische" Begrifflichkeit allgemeiner, ethischer Normierung betont wird.

Grundtvig will "das Gegenwärtige, wie es wirklich ist" (Den danske Stats-Kirke upartisk betragtet, 1834 VU III 321). Die erfüllte Lebenswirklichkeit des Einzelnen, jedes Menschen, sah Grundtvig bedroht durch das "Phantom der Abstraktion des Denkens", durch das "Falsum der Objektivität«, weil das Recht des Einzelnen auf selbsttätige Bewegung, die allein Wirklichkeit erzeugt, in einem solchen Denken bestritten wird. Die Furcht vor der Manipulierbarkeit des Menschen durch willkürliche Systementwürfe in einer Sprache, die bewusst unverständlich gehalten ist, treibt Grundtvig dazu in aller polemischer Schärfe gegen die "Krähensprache der Wissenschaft « zu kämpfen.

Sie wird für ihn von Professoren und Dozenten, Politikern und Staatsdienern gebraucht um das Volk zu "entmündigen" und es "geistig totzuschlagen".

Das einzig wahrhaft Soziale liegt in der Anerkennung jedes einzelnen Menschen. Allein jedoch durch das "lebende Wort " in seiner Abhängigkeit vom absoluten Guten wird der einzelne Mensch fähig, frei von Zwang in der und für die Gemeinschaft zu leben.

2. Kierkegaard hingegen, verwöhnter Kapitalistensohn und Bonvivant der Grossstadt, hatte, vor allem in seiner Jugend, im Gegensatz zu Grundtvig keine unmittelbare Beziehung zu anderen Ständen. Er erarbeitete sich sein Menschenbild aus der phänomenologischen Beobachtung seiner selbst und seiner Umwelt. Er hatte einen anderen Weg als Grundtvig vor sich, bedingt durch seine Erziehung und die entscheidende Prägung seiner Psyche durch die abnormen Bedingungen und Konstellationen in seinem Elternhaus. Aus ihnen heraus musste er einen Weg zu sozialer Einsicht und der Einheit von Volk und Individuum (die harmonische Gemeinschaft erst ermöglicht) finden. Da gab es keine natürlichen unmittelbaren Eindrücke, wie Grundtvig sie hatte, sondern allein Abstraktionen vom Menschlichen, die es unter Mühen und Qualen zu überwinden galt.

Seinen Weg erkämpfte er so auf ganz andere Weise als Grundtvig. Das Ziel war dasselbe: Die Freiheit des selbstbewussten Einzelnen, der sich seiner Hingabebereitschaft für die "Sache", die immer nur eine Sache der Gemeinschaft sein kann, bewusst ist. 
In kurzen Zügen seien die Stufen des intentionalen Bewegungsablaufes skizziert, in denen der Einzelne sich selbst als ein "organisches Glied " eines "organischen Ganzen", wie Kierkegaard sagt, nämlich seiner Gemeinschaft, hervorbringt.

Es ist der Einzelne, der einen Einsatz für eine bestimmte Sache, "det bestemte Noget", wagt und so die Sache des Guten, die Dinge der Welt positiv in Bewegung bringt. In der Hingabe an das Gute wird "berechtigte Wirklichkeit" konstituiert. Die Arbeit an der "Sache" ist immer auch die Sache des anderen Menschen, "des Armen, des Kranken, des Verbrechers ". Nur der Einzelne vermag, in Anerkennung der gegebenen Selbständigkeit jedes anderen Menschen, nach Kräften alles zu tun, um "die gegebene Selbständigkeit des anderen zu bewahren und ihn nach Kräften zu fördern" (VII 220). Der Einzelne, der den Mut hat, gegen Ausnutzung und Unterdrückung durch verrottete Gesinnung anzugehen, er allein ist die Quelle der Bewegung, hin zu einem neuen Menschen:

"Von Natur aus ist jeder Mensch darauf angelegt, ein Denker zu werden" (F.u.Z., S. 69). Ich fordere von jedem Menschen, er solle nicht so unmenschlich von sich denken, als ob er nicht in jene Paläste treten dürfte, in denen nicht bloss die Erinnerung an die Erkorenen wohnt, in denen sie vielmehr selber wohnen" (F.u.Z., S. 69).

Der erbitterte Abwehrkampf gegen die Metaphysik Hegels führte Kierkegaard zu dem Punkt im System Hegels, der ihm die offene Stelle, den "wunden Punkt" bot, der ihn das System aus den Angeln heben liess.

Dieser Punkt ist der Anfang der Logik Hegels. Dort nämlich, enthüllt Hegels Sprache, geschieht Bewegung "von allein". Ein unbestimmtes "Es" setzt sich "von allein". Es ist nicht die Rede von einem "Wie" oder "Wer" der Bewegung. Sie geschieht abstrakt, unbezogen, "von allein «! Im reinen Denken des "objektiven " Systems geschieht etwas, was dem einzelnen Menschen, von dem abstrahiert wird, nur als Wunder begegnen kann, ein Wunder, das er nur anzuschauen vermag. Niemand weiss, wie Bewegung geschieht, niemand, wer ihr Träger ist.

Für Kierkegaard ist die "von allein " sich vollziehende Bewegung des unbestimmten "Es" schimärisch, unwahr. Für ihn geschieht Bewegung nur in Freiheit. Und nur ein Mensch, der ein- 
zelne Mensch nämlich, "jeder Mensch", wie er hundertfach betont, kann Träger dieser Freiheit sein.

In der Beantwortung dieser Frage nach dem "Wie" und dem "Wer" dieser Bewegung entlarvt Kierkegaard Hegels Betrug am Menschlichen. Er zeigt den schimärischen Charakter dieser ominösen Allein-Bewegung als Betrug einer Gruppe von Menschen, die sich das System als Schutzschild vorhalten, um hinter ihm, als Einzelne unverantwortlich, ihr wahres Tun und Denken zu verbergen. Ihr wahres Tun weist sich als Ausbeutung und Unterdrückung der weniger Klugen, weniger Geschickten aus, die sie zu manipulieren und auszunützen trachten.

Als Funktionäre eines Systems, das den schützenden Eisenschirm des "absoluten Geistes" bietet, formieren sie sowohl den preussischen Staatapparat als auch den Menschenvernichtungsund Ausrottungsapparat des Nationalsozialismus. Nicht ausgenommen sind die herrsch- und machtsüchtigen Systeme aller christlichen Kirchen der Geschichte.

Das Beute-Machen, Zupacken, Angreifen des Anfangs in der Logik Hegels (das Wort "Anfang" wies noch alle diese Bedeutungen auf) zeigt für Kierkegaard überdeutlich, dass diesem System und seinem Anfang entgegengewirkt werden muss. Die Entmündigung des Einzelnen, im Namen eines Systems, das vorgibt, für den Einzelnen die Bewegung vollbringen zu können, ja vorgibt, sie immer schon vollzogen zu haben, zerstört und zertritt "die Selbständigkeit, die jedem Menschen gegeben ist ". Sie liefert ihn der Ausbeutung, Ausnützung, Manipulierbarkeit aus.

Eine der erregendsten Erkenntnisse liegt für Kierkegaard in der Entdeckung der Tatsache, dass die Gewalt, die gegen den Einzelnen im Laufe der Geschichte geübt wurde, von der Kirche im Gewand des Staates ausging. Der Staat, als verkleidete Kirche, die Kirche als verkleideter Staat, haben in ihrer unaufhörlichen Verknüpfung durch die Jahrhunderte hindurch zum Beispiel das Wort "lidelse" (Leiden), das eine freie, selbständige Bewegung bedeutete, zugunsten des (herkunftmässig verschiedenen) Wortes "lede" (Leid) verdrängt. "Lede" bedeutet ursprünglich Widerwärtigkeit, hartes Schicksal, das von aussen kommt. Nun schaffte die Kirche, d.h. der Staat, die positive Bedeutung ab, um seinem Kirchenvolk "einzubilden ", dass die Bibel das Erdulden von Unterwerfung, Zwang und Ausnutzung fordere. Kierkegaard 
hat diesen auch sprachlichen Betrug der Kirche aufgedeckt und die Begriffe "lidelse" und "lede" wieder geschieden.

"Diese unglücklichen Millionen, die man um ihr Geld (und ihr Leben) betrogen hat und betrügt, die man als Mittel benutzte (Arbeitskraft) und sie um das Ewige betrog, indem man ihnen Lügenmärchen erzählte" (XIV, 180): "Profitten er Sandhed i Kirkehistorien." Lügen und Märchen wurden dem Volk erzählt, um den Kirchenfürsten ihre fünf Mätressen, mit je einem Schlossflügel und fürstlicher Ausstattung, zu ermöglichen.

Dieser schändliche Betrug am Menschen, der sich fortschreitend in der Geschichte modifiziert, aber nicht wandelt, setzt sich für Kierkegaard im kapitalistischen Gebaren des Bürgertums fort. "For Penge-Fordelens og den sandselige Magts Skyld" spielt man den braven Bürger, um seinen Mitmenschen, den Armen auszunützen, zu hintergehen und zu betrügen.

Das gesamte Werk Kierkegaards ist durchsetzt von dem Ausdruck "jeder Mensch", denn für ihn gibt es in seinem Werk keine Klassenunterschiede, und so spricht er auch den "menige mand " als den an, der als einziger weiss, was Ausbeutung und Betrug von oben her bedeutet.

"Thi ikke sandt. Du menige Mand, det kan Du meget godt forstaae, jeg mener, at just Du kan langt lettere og bedre forstaae det end fordærvet Fornemhed, ikke sandt, dette kan Du ypperligt forstaae?" (XIV, 97).

Die Kritik am gesellschaftlichen Überbau, dem es lediglich um den eigenen Vorteil, den Profit, wie Kierkegaard sagt, geht, wird bei Kierkegaard besonders in seiner umfangreichen Sprachkritik deutlich. Das Dienstmädchen und der Landsoldat sind es, die nicht nur "die innere Ursprünglichkeit der Sprache" besitzen, sondern auch die Kraft der Überwindung von Gedankenlosigkeit, Geistreichigkeit, die gesellschaftliche Ausschaltung des Überbaus. "Såsnart der derimod kommer et Menneske som fører Primitivitet med sig, så han altså ikke siger: han må tage Verden som den er...", "... så bliver alt til Liv" (XI 21 A 46).

Gemeint sind diese Worte gegen die sozialen Zwänge der Oberschicht seiner Zeit, der es ausschliesslich um die Erhaltung ihrer Macht- und Herrschaftsposition gehen musste, die nur einem Gebot folgte, um oben zu bleiben und zu herrschen: Anpassung. "Boshaft wie die Sprache ist der Mensch, ungenau, unwahr" 
(VII, 271), um den geistig Unterlegenen zu hintergehen, auszunützen und zu betrügen (XII, 48). "Jo mere jeg forfølger Sagen, jo mere seer jeg at Forvirringen stikker ikke blot i Danmark, ikke blot i Protestantismen, og ikke blot i Christenheden, men at den stikker i Menneske-Naturen og dermed i det Tyve-Sprog som (vi) Mennesker tale (Pap. XI, 2 a 37).

Der neue soziale Mensch wird durch tägliche Selbstüberwindung um der gemeinsamen Sache willen erarbeitet. Die Spannung, die der Mensch täglich abzubauen hat, ermöglicht menschliches Wachstum, als lebenserhaltende Basis jeder harmonischen Gesellschaft.

Ohne hier das gesamte Problem der Kierkegaardschen Kommunikationstheorie auch nur andeuten zu können, muss doch die Bedeutung ihrer Grundzüge für die modernen sozialen Vorstellungen Kierkegaards festgestellt werden: Kierkegaards Einsicht, dass ein Miteinander-Sein ohne den Willen, den anderen zu fördern und ihm zu helfen, unsozial und deshalb unmenschlich und sinnlos ist, führt ihn darauf, eine völlig neue Kommunikationstheorie auszuarbeiten. Kommunikation ist nur als ethisch verantwortbare, gezielt positiv wirkende, lebendig. Das Wichtigste ist die Förderung der Eigenkräfte des anderen Menschen. Die objektive Mitteilung des Wissen ist gleichgültig (ligegyldig Viden), direkt, der eines Computers vergleichbar. Wissensvermittlung, die tote Information gibt, kann nicht als Kommunikation bezeichnet werden. "Nu har vi tænkt Meddelelsen Dialektik igennem som Viden og see at den er afskaffet. Nu følger et nyt Begreb af Meddelelsen" (VIII, 2 B 81, 12).

"Egenkærlighedens udryddelse overvinder alt, hvad der stiller sig hindrende $\mathrm{i}$ vejen for frembringelse af frugt i kommunikationen. $\mathrm{Og}$ det er en "uhyre Anstrengelse" at arbejde på den måde for samfundets fælles bedste."

3. Grundtvig und Kierkegaard haben zwar nicht in gleicher Weise zu den Tagesthemen öffentlich Stellung genommen, aber beide haben entschieden auf den Wandel der Bewusstseinsstruktur aller Menschen, als Grundlage effektiver Erneuerung hingearbeitet und damit die Entwicklung des heutigen staatsbürgerlichen Bewusstseins ermöglicht. All die bekannten Forderungen des "moderne gennembrud" waren die Forderungen Grundtvigs und 
Kierkegaards. Und sowohl Grundtvig als auch Kierkegaard wurden von den Männern des "moderne gennembrud " gekannt und gelesen. Die Verwirklichung sozialer Reformen, wie sie in Nordund Westeuropa stattgefunden haben, war nur möglich, weil diese Männer das Problem des einzelnen, selbstverantwortlichen Menschen zum Thema ihrer leidenschaftlichen Arbeit gemacht haben-, und zwar in einer radikalen, alle bisherigen Vorstellungen umstürzenden Weise. Durch ihren Einfluss befahigten sie auch die bürgerlichen Kräfte, endlich soziale Forderungen zu verstehen, sich ihnen anzuschliessen und so Reformen zu ermöglichen.

Grundtvig und Kierkegaard erarbeiteten die phänomenologischen Grundlage eines jeden sozialen Weltbildes. So ist der Gedanke, dass "jeder Mensch" (einer der häufigsten Begriffe Kierkegaards) schöpferisch sein kann und es - will er sich nicht ohne Sinn gegen die Gemeinschaft und sich selber wenden - auch sein sollte, schon vor Marx und erst recht vor allen Heutigen, die sich, wie Kierkegaard sagt, "in geschwätziger Nachäffung " ergehen, schon bei Grundtvig und Kierkegaard gedacht - bei Kierkegaard in endgültiger Klarheit.

Es sollte unserer Zeit auch deutlich werden, dass Kierkegaard sowie Grundtvig die absolute Abhängigkeit des modernen abendländischen Denkens (auch die des modernen Kommunismus) vom ursprünglich christlichen Denken sichtbar gemacht haben.

Grundtvigs und Kierkegaards Kraft gehörte der Erarbeitung der Grundlagen einer neuen Menschen- und Weltvorstellung. Sie gehörte der Frage, wie der Mensch der drohenden Gefahr des von allem Humanen gelösten rational-technischen Denkens und der des unbezogen-metaphysisch-idealistischen Denkens entgehen könnte. "Al Fordærvelse vil tilsidst komme fra Naturvidenskaben " (Pap. VII, I A 186, 123).

Die Wurzel ihrer Gemeinsamkeit ist der Kampf um die Grundlagen und Bedingungen der Selbstbestimmung des Menschen.

\section{Abkürzungen:}

V.U. = N.F.S. Grundtvig: Værker i Udvalg v. G. Christensen og Hal Koch, $1942 \mathrm{ff}$.

DV = Danne-Virke I (= VU II): Om Dansk Poesi, Sprog og Historie. 
SiU $=$ N. F. S. Grundtvig: Skrifter i Udvalg ved Kaj Thaning, 1965.

F.u.Z. = Søren Kierkegard: Furcht und Zittern in: Gesammelte Werke, Düsseldorf-Köln 1952 ff. IV (Eug. Diederichs Verlag.

I-XIV = Søren Kierkegaards Samlede Værker, udg. af A. B. Drachmann, J. I. Heiberg, H. O. Lange, $1901 \mathrm{ff}$.

Pap. Iff = Søren Kierkegaards "Papirer « udg. af P. A. Heiberg og V. Kuhr, 1909 ff.

(Furcht und Zittern $=$ Frygt og Bæven, hier ins Dänische zurückübersetzt, mit moderner Orthographie). 